\title{
Somatic BRCA1 mutations in clinically sporadic breast cancer with medullary histological features
}

\author{
Markus Rechsteiner ${ }^{1} \cdot$ Konstantin Dedes $^{2} \cdot$ Daniel Fink $^{2} \cdot$ Bernhard Pestalozzi $^{3} \cdot$ Bettina Sobottka $^{1} \cdot$ Holger Moch $^{1}$. \\ Peter Wild ${ }^{1} \cdot$ Zsuzsanna Varga $^{1}{ }^{10}$
}

Received: 30 October 2017 / Accepted: 13 February 2018 / Published online: 17 February 2018

(c) The Author(s) 2018. This article is an open access publication

\begin{abstract}
Background The role of somatic BRCA1/2 gene mutations in breast cancer is getting increasing attention in view of hereditary disease. The medullary phenotype and triple negative intrinsic subtypes are often, but not exclusively encountered in $B R C A 1$ germline mutated breast cancer, whilst for $B R C A 2$, no association to specific histological features are known. In this study, we addressed the relationship between morphological medullary phenotype and BRCA1/2 somatic mutations in breast cancer without known positive family anamnesis.

Methods 32 clinically sporadic breast cancers with medullary features were analyzed for somatic BRCA1/2 mutations (all coding exons) with next-generation sequencing technology. Paraffin-embedded formalin-fixed breast cancer samples from all patients were analyzed.

Results Three of 32 tumors (9\%) had pathogenic (ARUP class-5) BRCAl gene alterations. Two of these pathogenic variants exhibited deletions leading to frameshift mutations (p.Glu23fs, p.Val1234fs), and the remaining single-nucleotidvariant resulted in premature STOP codon (p.Glu60Ter). In one patient, the same pathogenic BRCAl mutation was detected (p.Glu23fs) in normal breast tissue. Retrospective follow-up in two patients revealed a positive family history for breast cancer and consecutive germline mutation testing confirmed presence of BRCA1 mutations. No somatic pathogenic BRCA2 mutations were detected.

Conclusions BRCAl mutation testing may be useful in clinically sporadic breast cancer patients with medullary features to identify potential mutation carriers independently from intrinsic molecular subtype. Formalin-fixed paraffin-embedded cancer tissue can undergo testing within a routine molecular-diagnostic setting as a clinical BRCAl/2 mutation screening strategy.
\end{abstract}

Keywords Sporadic breast cancer $\cdot$ Medullary features $\cdot$ BRCA1/2 mutation $\cdot$ NGS

\section{Introduction}

Assessment of BRCA1/2 gene mutation status from formalin-fixed paraffin-embedded (FFPE) tissue became a routine procedure for patients with high-grade serous ovarian cancer, as patients with evidence of such mutations are eligible for therapies including the PARP inhibitor olaparib

Zsuzsanna Varga

zsuzsanna.varga@usz.ch

1 Department of Pathology and Molecular Pathology, University Hospital Zurich, 8091 Zurich, Switzerland

2 Department of Gynecology, University Hospital Zurich, Zurich, Switzerland

3 Clinic for Oncology, University Hospital Zurich, Zurich, Switzerland
(Hennessy et al. 2010; Mafficini et al. 2016; Moschetta et al. 2016; Muggia 2009; Oza et al. 2015). The role of BRCA1/2 gene mutations in patients with breast cancer is also getting more attention, with genetic counseling in view of a hereditary disease becoming a highly demanding field in patient care (Farrugia et al. 2008; Gonzalez-Angulo et al. 2011; Gross et al. 2016; Kwon et al. 2010a, b). The majority of $B R C A 1$ mutated breast cancers are so-called "triple negative" or of "basal-type". In contrast, not all "triple negative" breast cancer patients have a germline BRCA1/2 mutation. The indication for BRCA $1 / 2$ mutation testing is mainly based on clinical criteria, other than on histomorphological features (Dabbs 2012; Lakhani et al. 2012; Lips et al. 2017).

The medullary phenotype of breast cancer, which is often but not exclusively encountered in $B R C A 1$ germline mutation carriers, cannot reliably be used as an indication 
for genetic testing. (Dabbs 2012; Lakhani et al. 2012; Lips et al. 2017). The current WHO classification on breast cancer defines medullary differentiation as invasive high-grade carcinomas exhibiting various amounts of lymphocytic infiltration typically lacking in situ components and showing sharply demarcated edges towards the tumor periphery (Dabbs 2012; Lakhani et al. 2012; Lips et al. 2017). Overall survival of typical and atypical medullary breast carcinomas seem to be quite similar to each other, however, prognostic difference to ductal non-special type (NST) breast cancer is controversially reported in the literature (Dabbs 2012; Lakhani et al. 2012; Lips et al. 2017) (Mateo et al. 2016; Mavaddat et al. 2012). In case of BRCA2 mutations in breast cancer, suggestive morphological and prognostic features are even more unspecific and thus less helpful. A wide range of histological subtypes, mainly a luminal hormone receptor positive phenotype, can be seen in breast cancer patients with BRCA2 germline mutation (Dabbs 2012; Lakhani et al. 2012; Lips et al. 2017).

In this study, we explored the relationship between a medullary phenotype in a series of breast cancer patients without known positive family history and $B R C A I / 2$ mutation status with next-generation sequencing (NGS) technology. We analyzed $B R C A 1 / 2$ mutation status in 32 breast cancer patients with medullary features (Fig. 1). The thus found pathogenic mutations were retrospectively compared with non-tumorous tissue and/or available long-term follow-up data.

\section{Materials and methods}

\section{Breast cancer patients}

All tissue samples were retrospectively retrieved from the archives of the Department of Pathology and Molecular Pathology, University Hospital Zurich, Switzerland, encompassing a period of 1994-2015. We identified 32 breast cancer cases displaying medullary histological features as defined in the WHO 2012 as follows: all tumors had some or all of the following features as sharp circumscription or pushing peripheral areas, syncytial growth pattern, mainly high-grade nuclear morphology and at least focal prominent stromal and intratumoral lymphocytic infiltration (Figs. 2, 3). Patients' age varied from 31 to 85 years (mean age 52.3 years). Tumor size varied from 1.2 to $6.5 \mathrm{~cm}$ (mean tumor size $2.44 \mathrm{~cm}$ ). 16 of 32 cases (50\%) were negative for estrogen and progesterone receptors and also for Her2 (triple negative intrinsic phenotype), 3 of 32 cases (9.3\%) were Her2 positive and 13 of 32 cases (40.7\%) were hormone receptor positive and Her2 negative. All patients underwent either mastectomy or local wide excision with axillary lymph node dissection (Table 1). There was no history of ovarian cancer in this cohort, three patients had benign ovarian cysts including also one mature teratoma.

The study is a part of a retrospective larger breast cancer study previously approved by the Ethical Committee
Fig. 1 Flow chart of study design

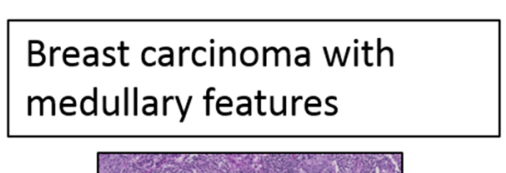

No information on positive family history

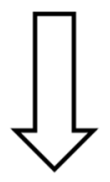

Paraffinblock with at least $50 \%$ tumor tissue

Next Generation Sequencing (NGS) using Oncomime BRCA $1 / 2$ assay
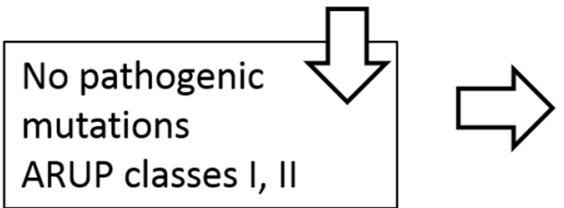

No further search in clinical charts

\begin{tabular}{|l|}
\hline Pathogenic \\
mutations \\
ARUP classes III, IV, V
\end{tabular}

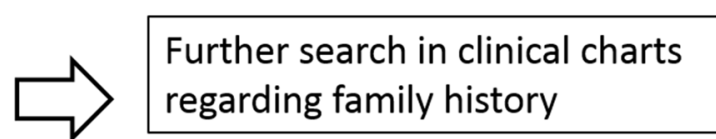


Fig. 2 Histological appearance of breast carcinoma with medullary features. a, b Low-power view of an invasive breast cancer with medullary features infiltration, serpentine-syntitial like tumor cell formation and sharp demarcation to tumor periphery, hematoxylin and eosin stain showing abundant lymphocytic
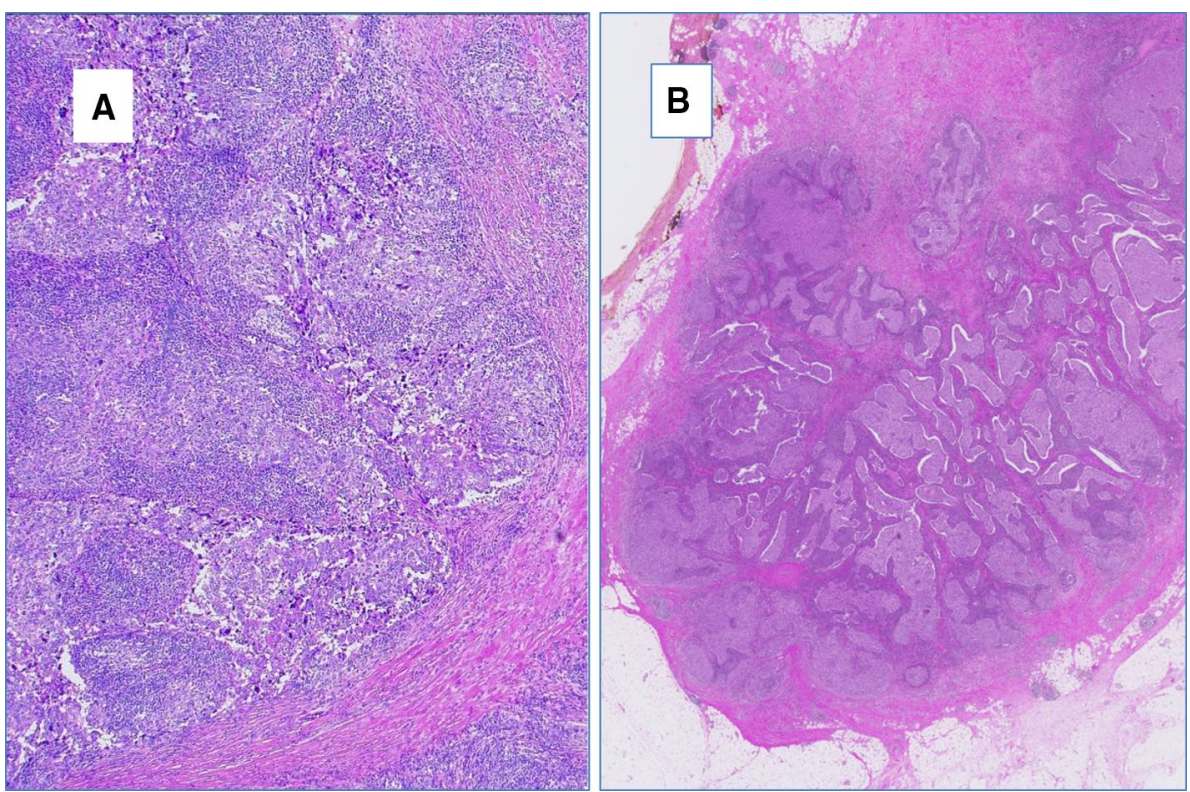

Fig. 3 a, b High-power view of an invasive breast cancer with medullary features. Syntitial tumor cell formation exhibiting high nuclear polymorphism and mitotic figures. Hematoxylin and eosin stain
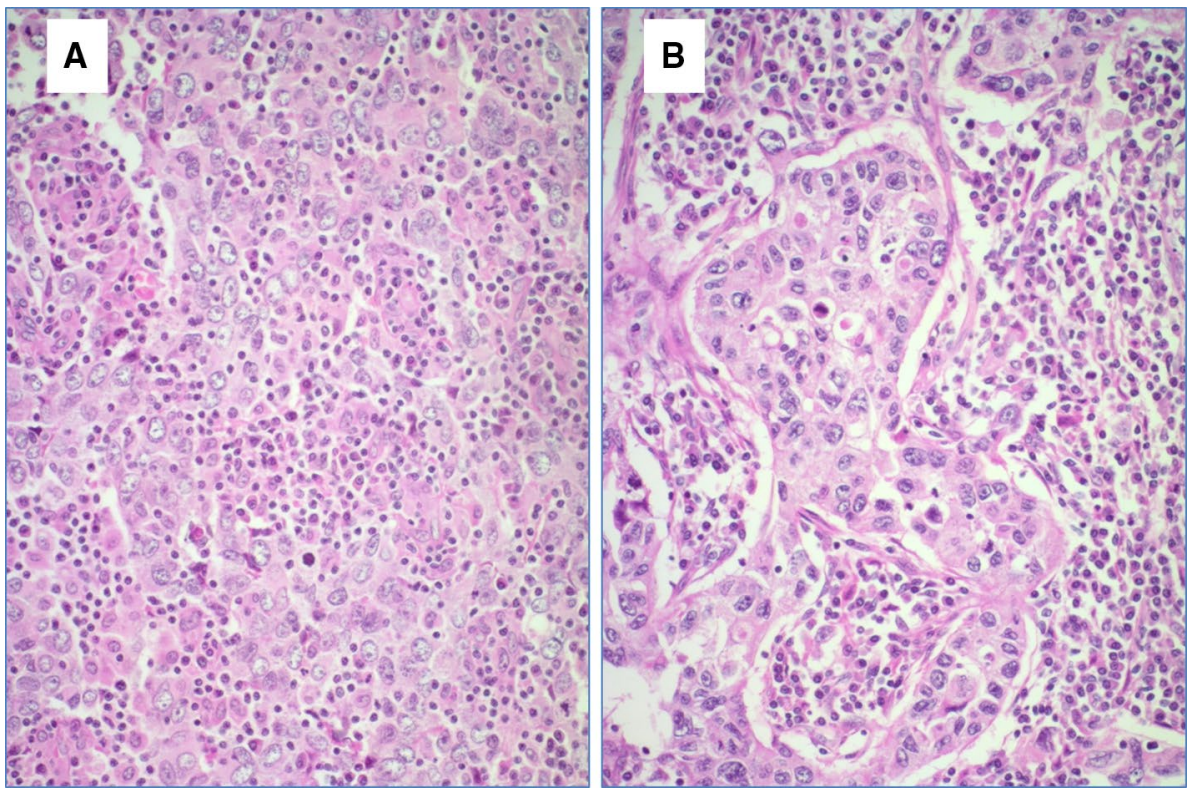

of the Canton Zurich (KEK-ZH-2012-553). For selected cases, required by the ethical approval, informed consents were obtained. All cases enrolled into the study cohort were anonymized for the study.

\section{Determination of hormone receptors and Her2 status}

The hormone receptors (estrogen, ER and progesterone, PR) were determined in all cases using routine antibodies and pretreatment conditions. HER2 status was determined using the DAKO Herceptest, the Ventana CB11 and Ventana 45A antibodies. Manual pretreatment protocols or semi-automatic and automatic benchmark systems have been used. Detailed technologies for ER/PR and HER2 status evaluation have been published, previously (Varga et al. 2013, 2014).

\section{Next-generation sequencing (NGS)}

For all NGS assays, representative cancer areas for the microdissection and DNA isolation were selected and marked by Z.V. on a freshly cut hematoxylin-eosin (HE) section. The marked tumor area was punched (length 2-4 $\mathrm{mm}$, diameter $0.6 \mathrm{~mm}$ ) from the paraffin block and DNA was isolated using a Promega DNA purification kit (Promega, Wisconsin, USA). Isolated genomic DNA 
Table 1 Clinico-pathological features of the cohort

\begin{tabular}{|c|c|c|c|c|c|c|c|c|}
\hline Case number & Histological diagnosis & Age (years) & Grading & $\begin{array}{l}\text { Tumor } \\
\text { size }(\mathrm{cm})\end{array}$ & ER & PR & $\begin{array}{l}\text { HER2 } \\
\text { IHC }\end{array}$ & $\begin{array}{l}\text { HER2 } \\
\text { FISH }\end{array}$ \\
\hline 1 & NST with medullary features & 39 & G3 & 2.4 & $100 \%$ positive & $100 \%$ positive & Score 0 & Not amplified \\
\hline 2 & NST with medullary features & 85 & G3 & 1.7 & $5 \%$ positive & $5 \%$ positive & NA & Not amplified \\
\hline 3 & NST with medullary features & 57 & G3 & 1.9 & Negative & Negative & Score 0 & NA \\
\hline 4 & NST with medullary features & 50 & G3 & 2.2 & Negative & Negative & Score 0 & NA \\
\hline 5 & NST with medullary features & 52 & G3 & 1.2 & Negative & Negative & NA & Not amplified \\
\hline 6 & NST with medullary features & 39 & G3 & 4 & Negative & Negative & NA & Not amplified \\
\hline 7 & NST with medullary features & 31 & G3 & 2 & Negative & Negative & $\mathrm{IHC} 0$ & NA \\
\hline 8 & NST with medullary features & 53 & G3 & 1.2 & Negative & Negative & Score $3+$ & Amplified \\
\hline 9 & NST with medullary features & 69 & G3 & 2.6 & Negative & Negative & NA & NA \\
\hline 10 & NST with medullary features & 59 & G3 & 2.6 & Negative & Negative & NA & NA \\
\hline 11 & NST with medullary features & 49 & G3 & 1.2 & Negative & Negative & NA & Not amplified \\
\hline 12 & NST with medullary features & 60 & G3 & 1.2 & $1 \%$ positive & $1 \%$ positive & NA & Not amplified \\
\hline 13 & NST with medullary features & 49 & G3 & 2.6 & $5 \%$ positive & $5 \%$ positive & NA & Not amplified \\
\hline 14 & NST with medullary features & 50 & G3 & 1 & Negative & Negative & NA & Not amplifed \\
\hline 15 & NST with medullary features & 48 & G3 & 2,5 & $60 \%$ positive & $2 \%$ positve & Score $1+$ & Not amplified \\
\hline 16 & NST with medullary features & 65 & G3 & 2.2 & $80 \%$ positive & Negative & Score $1+$ & Not amplified \\
\hline 17 & NST with medullary features & 49 & G3 & 0.9 & Negative & Negative & Score 0 & Not amplified \\
\hline 18 & NST with medullary features & 36 & G3 & 4.5 & Negative & Negative & Score 0 & Not ampified \\
\hline 19 & NST with medullary features & 56 & G3 & 2.3 & $100 \%$ positive & $10 \%$ positive & Score $1+$ & Not amplified \\
\hline 20 & NST with medullary features & 43 & G3 & 2.2 & $100 \%$ positive & $100 \%$ positive & Score $2+$ & Not amplified \\
\hline 21 & NST with medullary features & 45 & G3 & 3.5 & $100 \%$ positive & $50 \%$ positive & Score $2+$ & Not amplified \\
\hline 22 & NST with medullary features & 44 & G3 & 6.5 & Negative & Negative & Score $1+$ & Not amplified \\
\hline 23 & NST with medullary features & 65 & G3 & 6 & $100 \%$ positive & $90 \%$ positive & Score $1+$ & Not amplified \\
\hline 24 & NST with medullary features & 73 & G3 & 5 & $100 \%$ positive & $80 \%$ positive & Score $2+$ & Not amplified \\
\hline 25 & NST with medullary features & 55 & G3 & 1.2 & Negative & Negative & Score 0 & NA \\
\hline 26 & NST with medullary features & 36 & G3 & 3 & $20 \%$ positive & $10 \%$ positive & Score 0 & Not amplified \\
\hline 27 & NST with medullary features & 56 & G3 & 2.2 & $100 \%$ positive & $20 \%$ positive & Score $1+$ & Not amplified \\
\hline 28 & NST with medullary features & 45 & G3 & 2.6 & $100 \%$ positive & $80 \%$ positive & Score $3+$ & Amplified \\
\hline 29 & NST with medullary features & 54 & G3 & 1.2 & Negative & negative & Score $3+$ & Amplified \\
\hline 30 & NST with medullary features & 76 & G3 & 0.6 & Negative & Negative & Score $2+$ & Not amplified \\
\hline 31 & NST with medullary features & 47 & G3 & 2.2 & $100 \%$ positive & Negative & Score 0 & Not amplified \\
\hline 32 & NST with medullary features & 39 & G3 & 1.8 & Negative & Negative & Score 0 & Not amplified \\
\hline
\end{tabular}

was quantified using a fluorometric assay (Qubit, Thermo Fisher Scientific, Massachusetts, USA) and NGS libraries were amplified with the AmpliSeq Library Kit 2.0 and the Oncomine BRCA Assay (Thermo Fisher Scientific). Clonal amplification und sequencing was performed with Hi-Q chemistry on the Ion PGM platform according to manufacturer's requirements (Thermo Fisher Scientific). NGS data was analyzed with the Torrent Suite v5.0.3 and the Ion Reporter v5.0 including the Oncomine BRCA workflow. NGS run metrics (on target reads, mean depth, uniformity) are summarized in Table 2. NGS reads were aligned to the reference genome hg19/GRCh37 and the transcripts for BRCA1 (NM_007300.3) and BRCA2 (NM_000059.3). The detected variants were filtered to the coding exons and excluded if they were listed in the
commonSNP database (minor allele frequency $>1 \%$ ). Additionally, variants were excluded if they had variant allele frequencies $<4 \%$ and variant allele coverage $<50 \times$ (internal validation of sensitivity of AmpliSeq assays). To further validate the performance of the Oncomine BRCA panel, a mixing dilution experiment was performed confirming the sensitivity of the assay for SNVs and INDELs. After filtering, the variants were annotated to the COSMIC, dbSNP, and ARUP BRCA databases (http://arup. utah.edu/database/BRCA/). ARUP classification was done according to Plon et al. (2008): (1) (not pathogenic or of no clinical significance); (2) (likely not pathogenic or of little clinical significance); (3) (uncertain); (4) (likely pathogenic); and (5) (definitely pathogenic). To further classify the impact of unknown mutations on protein level, 


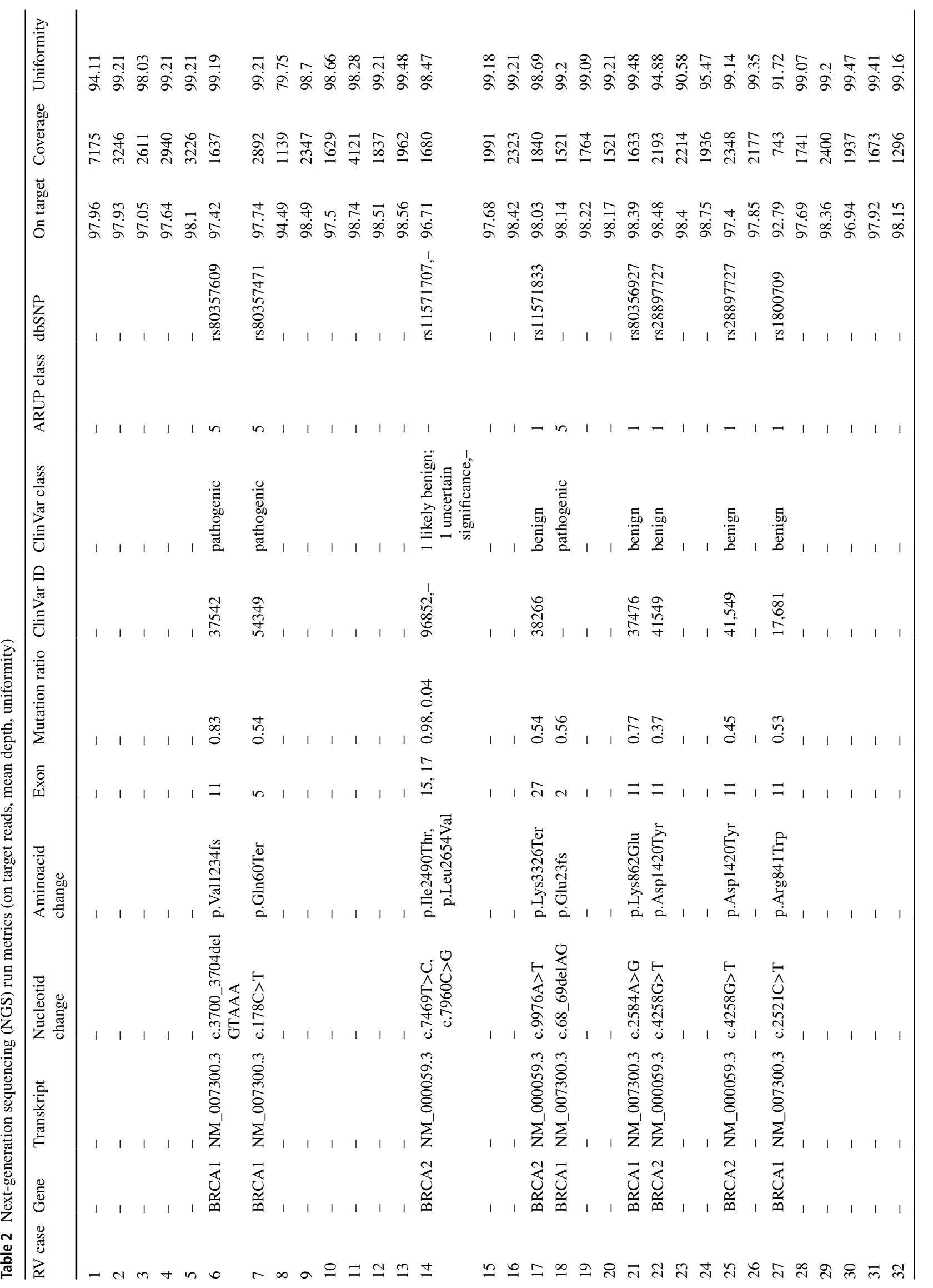


SIFT (http://sift.jcvi.org/) and PolyPhen (http://genetics. bwh.harvard.edu/pph/) were used.

\section{Results}

Three of 32 cases ( $9 \%$ ) had definitely pathogenic germline BRCA1 mutations (ARUP class 5) which lead either to a frameshift or a STOP codon in the protein (Table 3). No pathogenic somatic BRCA2 mutations were observed.

The first patient (no. 6) was diagnosed with breast cancer at an age of 40 years. NGS-based BRCA1/2 testing showed a 5-basepair deletion (c.3700_3704delGTAAA) in exon 11 in the BRCAl gene which lead to a frameshift at aminoacid position 1234 (p.Val1234fs). The mutation is registered in the databases ARUP, ClinVar (ID 37542) and dbSNP (rs80357609) to be pathogenic. A second independent NGS library was prepared and sequenced which successfully validated the BRCAl mutation p.Val1234fs. Retrospective follow-up search revealed a positive family history in this patient. Due to a contralateral breast cancer 20 years after the initial diagnosis, the patient underwent germline $B R C A 1$ mutation testing, revealing the same pathogenic mutation in BRCAl.

The second patient (no. 7) with a pathogenic BRCAl alteration had a nonsense mutation (c. $178 \mathrm{C}>\mathrm{T}$ ) in exon 5 in the $B R C A 1$ gene leading to a STOP codon in the protein (p.Gln60Ter). This mutation is as listed as well in the databases ARUP, ClinVar (ID 54349) and dbSNP (rs80357471) to be pathogenic. An independent NGS library was prepared and sequenced which successfully validated the
Table 3 Pathogenicitiy classification of detected mutation in the analyzed 32 cases

\begin{tabular}{|c|c|c|c|c|c|}
\hline RV case & I & II & III & IV & $\mathrm{V}$ \\
\hline 1 & - & - & - & - & - \\
\hline 2 & - & - & - & - & - \\
\hline 3 & - & - & - & - & - \\
\hline 4 & - & - & - & - & - \\
\hline 5 & - & - & - & - & - \\
\hline 6 & - & - & - & - & BRCA1: p.Val1234fs \\
\hline 7 & - & - & - & - & BRCA1: p.Gln60Ter \\
\hline 8 & - & - & - & - & - \\
\hline 9 & - & - & - & - & - \\
\hline 10 & - & - & - & - & - \\
\hline 11 & - & - & - & - & - \\
\hline 12 & - & - & - & - & - \\
\hline 13 & - & - & - & - & - \\
\hline 14 & - & $\begin{array}{l}\text { BRCA2: } \\
\text { p.Ile2490Thr }\end{array}$ & - & $\begin{array}{l}\text { BRCA2: } \\
\text { p.Leu2654Val }\end{array}$ & - \\
\hline 15 & - & - & - & - & - \\
\hline 16 & - & - & - & - & - \\
\hline 17 & BRCA2: p.Lys3326Ter & - & - & - & - \\
\hline 18 & - & - & - & - & BRCA1: p.Glu23fs \\
\hline 19 & - & - & - & - & - \\
\hline 20 & - & - & - & - & - \\
\hline 21 & BRCA1: p.Lys862Glu & - & - & - & - \\
\hline 22 & BRCA2: p.Asp1420Tyr & - & - & - & - \\
\hline 23 & - & - & - & - & - \\
\hline 24 & - & - & - & - & - \\
\hline 25 & BRCA2: p.Asp1420Tyr & - & - & - & - \\
\hline 26 & - & - & - & - & - \\
\hline 27 & BRCA1: p.Arg841Trp & - & - & - & - \\
\hline 28 & - & - & - & - & - \\
\hline 29 & - & - & - & - & - \\
\hline 30 & - & - & - & - & - \\
\hline 31 & - & - & - & - & - \\
\hline 32 & - & - & - & - & - \\
\hline
\end{tabular}


mutation. Subsequent germline $B R C A 1$ mutation testing showed the same pathogenic mutation in BRCAl.

The third patient (no. 18) had as a deletion (c.68_69delAG) in the BRCA1 gene, leading to a frameshift in the protein (p.Glu23fs). Subsequent NGS analysis of corresponding normal breast tissue revealed the same mutation, suggesting a hereditary disease. In this patient, there was no positive family history of breast cancer. Due to the young age (37 years at initial diagnosis) and the triple negative phenotype with medullary features, genetic counseling and testing was recommended to the patient at the weekly interdisciplinary tumor board. Four years after initial diagnosis, however, no records about germline $B R C A 1 / 2$ testing could be found.

Pathogenic mutations of the BRCA1 gene are illustrated in details in Fig. 4.
Fig. 4 Pathogenic mutations of the BRCA1 gene in patients 6 , 7 and 18

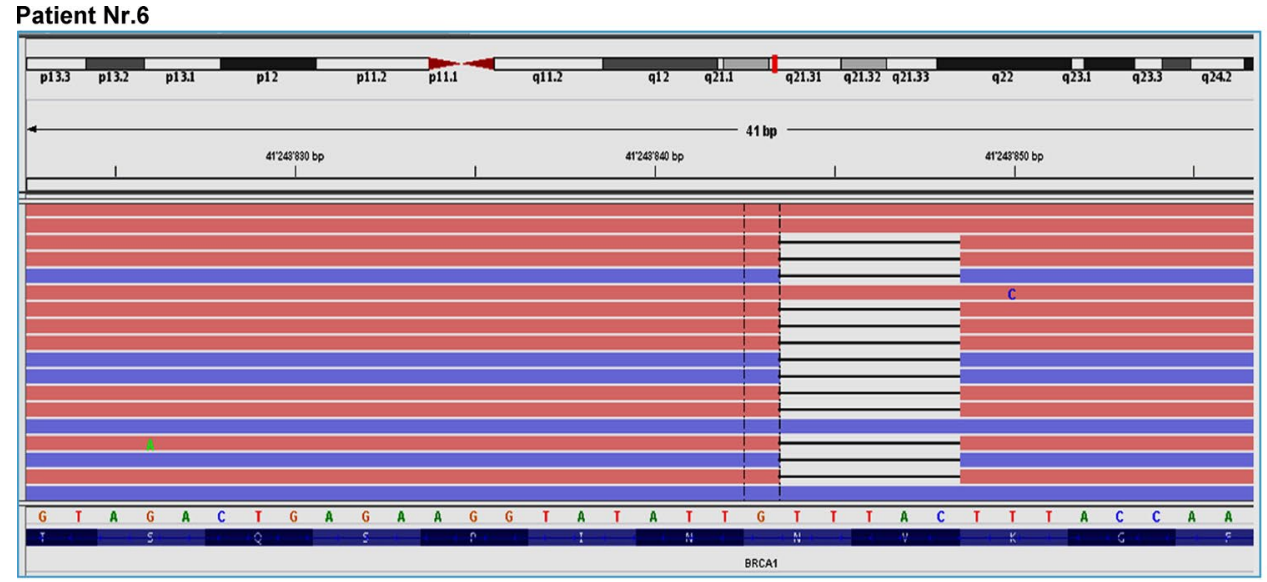

Patient Nr. 7.

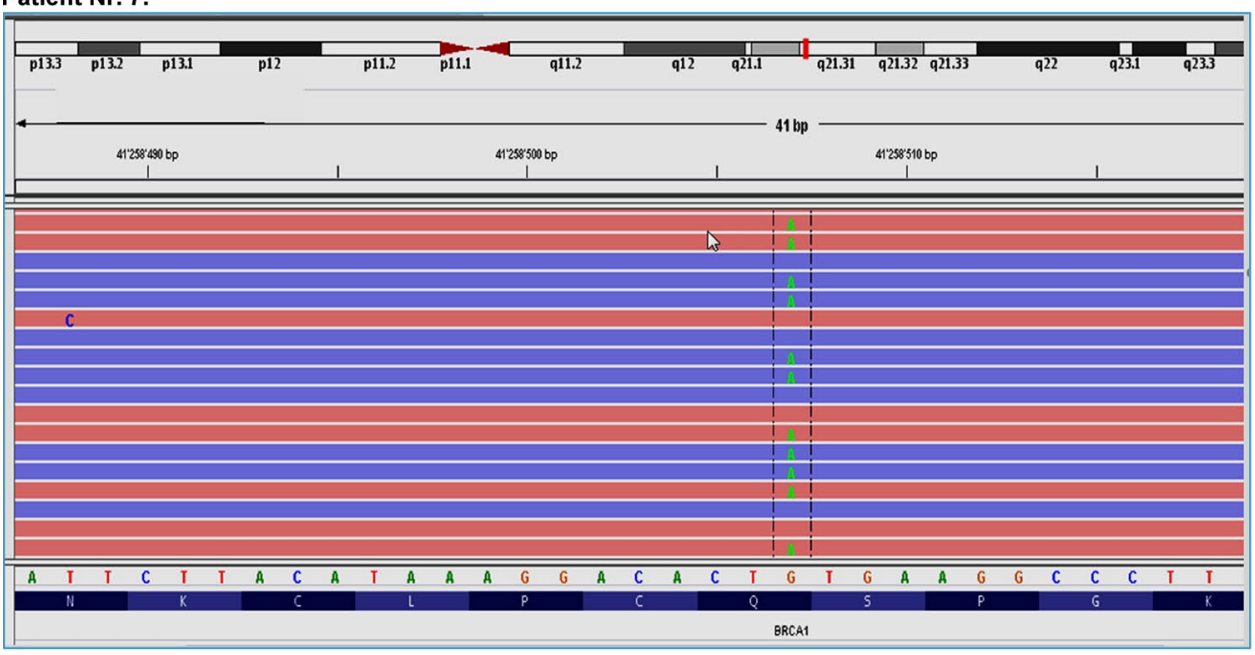

\section{Patient Nr.18}

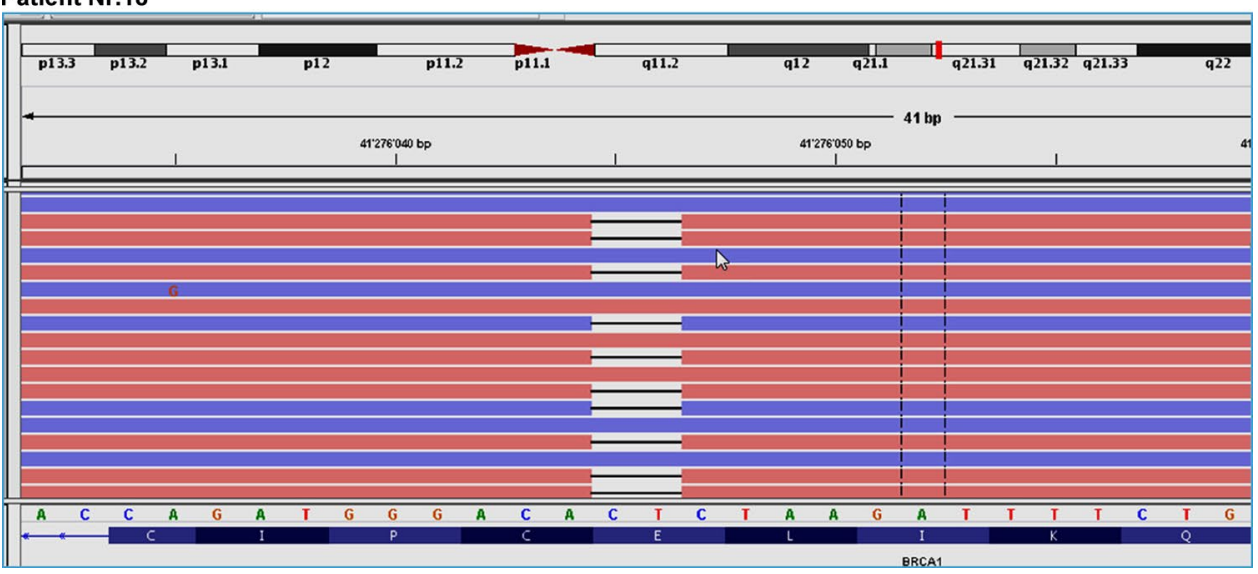


In six patients, benign, likely benign or mutations of unknown significance (VUS) were detected (ARUP class $1-3)$.

In one patient (no. 14), two SNVs were detected in exon 15 (p.Ile2490Thr) and 17 (p.Leu2654Val) of the BRCA2 gene. The mutation in exon 15 showed an allele frequency of almost $100 \%$, representing a homozygous SNP, which was further underlined by a dbSNP entry (rs11571707) with a minor allele frequency (MAF) of $1.9 \%$ in the human population. The same mutation is registered in ClinVar (ID 96852) with conflicting interpretation of clinical significance. However, these entries are benign (11 entries), likely benign (1 entry), and of uncertain significance ( 1 entry), suggesting a non-pathogenic impact. The second mutation in patient no. 14 was detected at low allele frequency of $4 \%$. Since this is at the limit of detection of our NGS system, a second independent NGS library was prepared and sequenced. The mutation p.Leu2654Val was successfully verified with an allele frequency of $5 \%$. Interestingly, this mutation is registered neither in COSMIC, dbSNP nor in the ARUP. The protein alteration prediction tools SIFT and PolyPhen revealed highly destabilizing (damaging) values of 0 and 0.87 , respectively. Therefore, the exon 17 mutation in BRCA2 (p.Leu2654Val) was classified as VUS.

One nonsense mutation (patient no. 17) was detected at the 3-prime end in exon 27 of the BRCA2 gene, leading to a STOP codon (p.Lys3326Ter). This mutation is registered in the ARUP and the ClinVar database and was suggested to be clinically not significant (ARUP class 1) (Farrugia et al. 2008; Tavtigian et al. 2008), and benign (ClinVar ID 38266), respectively. Additionally, the mutation is listed in the dbSNP database (rs11571833) with a minor allele frequency (MAF) of $0.4 \%$ in the human population. The mutation was not validated in an independent NGS library run, since it was assumed to be non-pathogenic.

Additional mutations which were assumed to be nonpathogenic according to ClinVar and ARUP entries were detected in patient no. 21 (BRCA1, p.Lys862Glu), no. 22 (BRCA2, p.Asp1420Tyr), no. 25 (BRCA2, p.Asp1420Tyr), and no. 27 (BRCA1, p.Arg841Trp). These mutations were not validated with an independent NGS run due to nonpathogenicity either.

\section{Discussion}

We performed BRCA1/2 testing by next-generation sequencing in clinically sporadic breast cancer patients with medullary like breast cancer and without known BRCAl and $B R C A 2$ mutations at presentation. Our study demonstrates that about nine percent of medullary like breast cancer patients without any known positive family history were
BRCAl gene mutation carriers, whereas no pathogenic somatic BRCA1/2 mutations could be found.

$B R C A 1 / 2$ mutations regained clinical attention, as patients with high-grade serous ovarian carcinomas with pathogenic $B R C A 1 / 2$ mutations displayed improved overall and recurrence-free survival if treated with platinumbased therapy in combination with PARP inhibitors such as olaparib (Hennessy et al. 2010; Mafficini et al. 2016; Moschetta et al. 2016; Muggia 2009; Oza et al. 2015; Kwon et al. 2010; Muggia et al. 2011). Patients with BRCA1/2 germline mutated serous ovarian cancers responded better to first-line chemotherapy in the metastatic setting in comparison with sporadic serous high-grade carcinomas (Hennessy et al. 2010; Mafficini et al. 2016; Moschetta et al. 2016; Muggia 2009; Oza et al. 2015; Kwon et al. 2010; Muggia et al. 2011). Furthermore, resistance to taxane containing regimens have been documented in serous high-grade ovarian carcinomas displaying BRCA $1 / 2$ germline mutations (Hennessy et al. 2010; Mafficini et al. 2016; Moschetta et al. 2016; Muggia 2009; Oza et al. 2015; Kwon et al. 2010; Muggia et al. 2011).

The role of pathogenic BRCA1/2 mutations in breast cancer is currently not linked to specific therapies and is rather restricted to the choice for genetic counseling (Chalasani and Livingston 2013). The need for genetic counseling including germ line BRCA1/2 testing in breast cancer patients is increasing, however, selection criteria remain mostly a positive family history additionally to breast cancers harboring a triple negative intrinsic phenotype (Kwon et al. 2010a, b; Chalasani and Livingston 2013). Specific histological signs only exist for germline mutations in BRCA1. Breast cancers arising in $B R C A 1$ germ line mutation carriers are mostly triple negative, of younger patients ( $<50$ years), high-grade and display so-called medullary features. Medullary-type breast cancers are characterized by dense lymphocytic infiltrate and pushing peripheral borders (Gonzalez-Angulo et al. 2011; Kwon et al. 2010a, b; Dabbs 2012; Lakhani et al. 2012; Chalasani and Livingston 2013). The frequency of BRCA1 gene allelic loss were reported to be more frequent in ER negative than in ER positive sporadic breast cancer cases (39 vs 12\%) (Rhiem et al. 2010). In unselected triple negative breast cancers, around $19 \%$ mutations were reported in the BRCA $1 / 2$ genes including also scattered somatic mutations (15\% in BRCA and $3.9 \%$ in BRCA2 genes) (Gonzalez-Angulo et al. 2011). Interestingly, there is lack of data on sporadic medullary carcinomas and its association with BRCA1 germline or somatic mutations. The $6.25 \%$ frequency of BRCA1 frame shift mutations in our study is lower than reported frequencies in unselected triple negative breast cancers or in sporadic cases or in serous high-grade ovarian carcinomas without family history varying from 19 to 28\%) (Gonzalez-Angulo et al. 2011; Rhiem et al. 2010) (Mafficini et al. 2016; Moschetta et al. 2016). 
Whether this lower frequency is due to the relatively small number of cases in our cohort or to the fact that medullary phenotype is alone not pathognomic enough to predict BRCA1 mutation status, needs to be validated in further studies. On the other hand, $40.7 \%$ of the cases were hormone receptor positive in our study, which is unusually high in comparison to classical triple negative phenotype of classical medullary breast carcinoma. The high proportion of hormone receptor positive cases might possibly reflect the histological variability of medullary differentiation in breast cancer and might also contribute to the low frequency of somatic BRCA1 mutations.

In selected triple negative breast cancer cases, $57 \%$ were found to have BRCA1 and 23\% BRCA2 germ line mutations (Gonzalez-Angulo et al. 2011).

The term BRCAness, defined as DNA repair loss in the BRCA1/2 genes without germ line mutations, resulting in the same function loss and inactivation the BRCA1/2 genes, has been conflictingly discussed and addressed in the literature (Muggia 2009; Lips et al. 2017; Muggia et al. 2011; Chalasani and Livingston 2013; Vollebergh et al. 2014). Probably, the choice of technology for assessing the DNA damage as MPLA, qPCR, IHC or aCGH methodologies resulted in non-standardized definitions of what consists of BRCAness damage (Chalasani and Livingston 2013; Lips et al. 2017). In two studies, breast cancers with BRCA1like signature, as defined as BRCAness of the BRCA1 gene were found in $18 \%$ of breast cancers and showing a better response to anthacyline-based or platin containing high-dose chemotherapies (Lips et al. 2017; Vollebergh et al. 2014). One further study reported a higher frequency of BRCA2like signatures in hormone receptor positive breast cancer cases and also a better response to neoadjuvant chemotherapy with anthracycline-based regiments (Lips et al. 2017). Which technology is the most reliable to predict clinical outcome or the indication to genetic counseling in cases with BRCAness evidence is not solved at the current time and needs clinical validation in further studies (Muggia 2009; Muggia et al. 2011; Chalasani and Livingston 2013).

The current technology of next-generation sequencing in paraffin-embedded, formalin-fixed material was first described in ovarian high-grade serious cancer both for somatic and germ line mutations, providing a sensitivity of $>90 \%$ after verifying the data with Sanger sequencing (Mafficini et al. 2016). This technology became meanwhile standard in somatic BRCA1/2 testing in ovarian cancer and was also the choice of methodology in our study. The classification system for detected mutations using NGS and Sanger sequencing was recently defined by Eccles (Eccles et al. 2015). A five-tier score system, ranging from non- or likely non-pathogenic mutations (classes $1 / 2$ ) through uncertain significance as class 3 to likely or definitely pathogenic mutations (classes 4/5) is linked to recommendations in terms of clinical management and to assessing risk situation of the given patient (Eccles et al. 2015). Scores 4/5 are recommended as high-risk patient with appropriate genetic counseling, whilst classes 1 and 2 should follow management based on family history alone. At the current time, no clear guidelines exist for the clinical management of genes of uncertain significance (class 3). (Farrugia et al. 2008; Plon et al. 2008; Tavtigian et al. 2008) (Eccles et al. 2015). In or study, we found three definitely pathogenic somatic mutations of the BRCA1 genes (class 5).

Based on the data in our cohort, medullary breast cancer phenotype without known family history in breast cancer, has a low frequency of somatic BRCA1 mutations, even though these mutations turned out to correlate with germ line mutations of the BRCA1 gene independently from other factors as younger age and triple negative intrinsic phenotype. These data might be of help in case genetic counseling in sporadic breast cancers with medullary features. On the other hand, medullary phenotype without family anamnesis did not have any class 4 or 5 somatic mutations in the BRCA2 gene, pointing out to the role of the BRCA1 gene only in breast cancer with medullary features. Our data need to be validated in further lager studies.

Acknowledgements The authors wish to acknowledge Annette Bohnert, André Fitsche, Christiane Mittmann, Peter Schraml (Tissue Biobank University Hospital Zurich), Martina Storz, Fabiola Prutek and Susanne Dettwiler for their technical assistance. Qing Zhong and Ulrich Wagner (HTG Laboratory) is thanked for their help with bioinformatics. Data from this paper were presented as poster at the USCAP Annual meeting in March 2017 in San Antonio.

Funding This work was supported in part by an innovation pool Grant to P.W.

\section{Compliance with ethical standards}

Conflict of interest The authors declare no conflict of interest.

Open Access This article is distributed under the terms of the Creative Commons Attribution 4.0 International License (http://creativeco mmons.org/licenses/by/4.0/), which permits unrestricted use, distribution, and reproduction in any medium, provided you give appropriate credit to the original author(s) and the source, provide a link to the Creative Commons license, and indicate if changes were made.

\section{References}

Chalasani P, Livingston R (2013) Differential chemotherapeutic sensitivity for breast tumors with "BRCAness": a review. Oncologist 18:909-916

Dabbs DJ (2016) Breast Pathology, 2nd edn, ISBN: 9780323462365 , ISBN: 9780323462372, ISBN: 9780323389617, Elsevier, p 848

Eccles DM, Mitchell G, Monteiro AN, Schmutzler R, Couch FJ, Spurdle AB, Gomez-Garcia EB, Group ECW (2015) Brca1 and brca2 
genetic testing-pitfalls and recommendations for managing variants of uncertain clinical significance. Ann Oncol 26:2057-2065

Farrugia DJ, Agarwal MK, Pankratz VS, Deffenbaugh AM, Pruss D, Frye C, Wadum L, Johnson K, Mentlick J, Tavtigian SV et al (2008) Functional assays for classification of brca2 variants of uncertain significance. Cancer Res 68:3523-3531

Gonzalez-Angulo AM, Timms KM, Liu S, Chen H, Litton JK, Potter J, Lanchbury JS, Stemke-Hale K, Hennessy BT, Arun BK et al (2011) Incidence and outcome of brca mutations in unselected patients with triple receptor-negative breast cancer. Clin Cancer Res 17:1082-1089

Gross E, van Tinteren H, Li Z, Raab S, Meul C, Avril S, Laddach N, Aubele M, Propping C, Gkazepis A et al (2016) Identification of brca1-like triple-negative breast cancers by quantitative multiplex-ligation-dependent probe amplification (MLPA) analysis of brca1-associated chromosomal regions: a validation study. BMC Cancer 16:811

Hennessy BT, Timms KM, Carey MS, Gutin A, Meyer LA, Flake DD, 2nd, Abkevich V, Potter J, Pruss D, Glenn P et al (2010) Somatic mutations in brca1 and brca 2 could expand the number of patients that benefit from poly (adp ribose) polymerase inhibitors in ovarian cancer. J Clin Oncol 28:3570-3576

Kwon JS, Gutierrez-Barrera AM, Young D, Sun CC, Daniels MS, Lu $\mathrm{KH}$, Arun B (2010a) Expanding the criteria for brca mutation testing in breast cancer survivors. J Clin Oncol 28:4214-4220

Kwon JS, Daniels MS, Sun CC, Lu KH (2010b) Preventing future cancers by testing women with ovarian cancer for brca mutations. J Clin Oncol 28:675-682

Lakhani SREISS, Tan PH, van de Vijver MJ (2012) Who classification of tumours of the breast, fourth edition 2012. International Agency for Research on Cancer, Lyon

Lips EH, Debipersad RD, Scheerman CE, Mulder L, Sonke GS, van der Kolk LE, Wesseling J, Hogervorst FB, Nederlof PM (2017) BRCA1-mutated estrogen receptor-positive breast cancer shows BRCAness, suggesting sensitivity to drugs targeting homologous recombination deficiency. Clin Cancer Res 23(5):1236-1241

Mafficini A, Simbolo M, Parisi A, Rusev B, Luchini C, Cataldo I, Piazzola E, Sperandio N, Turri G, Franchi M et al (2016) Brca somatic and germline mutation detection in paraffin embedded ovarian cancers by next-generation sequencing. Oncotarget 7:1076-1083

Mateo AM, Pezzi TA, Sundermeyer M, Kelley CA, Klimberg VS, Pezzi CM (2016) Atypical medullary carcinoma of the breast has similar prognostic factors and survival to typical medullary breast carcinoma: 3976 cases from the national cancer data base. J Surg Oncol 114:533-536
Mavaddat N, Barrowdale D, Andrulis IL, Domchek SM, Eccles D, Nevanlinna H, Ramus SJ, Spurdle A, Robson M, Sherman M et al (2012) Pathology of breast and ovarian cancers among brca1 and brca2 mutation carriers: Results from the consortium of investigators of modifiers of brca1/2 (cimba). Cancer Epidemiol Biomark Prev 21:134-147

Moschetta M, George A, Kaye SB, Banerjee S (2016) Brca somatic mutations and epigenetic brca modifications in serous ovarian cancer. Ann Oncol 27:1449-1455

Muggia F (2009) Brca-deficient animal models and cisplatin resistance. Ann Oncol 20:962

Muggia F, Safra T, Dubeau L (2011) Brca genes: lessons learned from experimental and clinical cancer. Ann Oncol 22(Suppl 1):i7-i10

Oza AM, Cibula D, Benzaquen AO, Poole C, Mathijssen RH, Sonke GS, Colombo N, Spacek J, Vuylsteke P, Hirte H et al (2015) Olaparib combined with chemotherapy for recurrent platinumsensitive ovarian cancer: a randomised phase 2 trial. Lancet Oncol 16:87-97

Plon SE, Eccles DM, Easton D, Foulkes WD, Genuardi M, Greenblatt MS, Hogervorst FB, Hoogerbrugge N, Spurdle AB, Tavtigian SV et al (2008) Sequence variant classification and reporting: Recommendations for improving the interpretation of cancer susceptibility genetic test results. Hum Mutat 29:1282-1291

Rhiem K, Todt U, Wappenschmidt B, Klein A, Wardelmann E, Schmutzler RK (2010) Sporadic breast carcinomas with somatic brca1 gene deletions share genotype/phenotype features with familial breast carcinomas. Anticancer Res 30:3445-3449

Tavtigian SV, Byrnes GB, Goldgar DE, Thomas A (2008) Classification of rare missense substitutions, using risk surfaces, with genetic- and molecular-epidemiology applications. Hum Mutat 29:1342-1354

Varga Z, Noske A, Ramach C, Padberg B, Moch H (2013) Assessment of her 2 status in breast cancer: overall positivity rate and accuracy by fluorescence in situ hybridization and immunohistochemistry in a single institution over 12 years: a quality control study. BMC Cancer 13:615

Varga Z, Tubbs RR, Moch H (2014) Concomitant detection of her2 protein and gene alterations by immunohistochemistry (ihc) and silver enhanced in situ hybridization (sish) identifies her2 positive breast cancer with and without gene amplification. PLoS One 9:e105961

Vollebergh MA, Lips EH, Nederlof PM, Wessels LF, Wesseling J, Vd Vijver MJ, de Vries EG, van Tinteren H, Jonkers J, Hauptmann $\mathrm{M}$ et al (2014) Genomic patterns resembling brca1- and brca2mutated breast cancers predict benefit of intensified carboplatinbased chemotherapy. Breast Cancer Res 16:R47 